\title{
Propriedades Físicas de Painéis Aglomerados Comerciais Confeccionados com Bagaço de Cana e Madeira
}

\author{
Cecília de Souza Freire, Danillo Wisky Silva, Mário Vanoli Scatolino, \\ Antônia Amanda da Silva César, Lina Bufalino, Lourival Marin Mendes \\ Departamento de Ciências Florestais - DCF, Universidade Federal de Lavras - UFLA
}

\begin{abstract}
RESUMO
As propriedades físicas de painéis aglomerados comerciais produzidos com bagaço de cana e madeira foram estudadas. Os testes de absorção de água e inchamento em espessura foram realizados para comparação entre os materiais estudados e entre os tipos de absorção de cada material: total, superficial e lateral. Nos testes de comparação entre painéis de bagaço de cana e painéis de madeira, foi observado que os primeiros apresentaram maiores valores de absorção de água, mas menores inchamentos em espessura. O aglomerado de bagaço de cana apresentou maiores valores de absorção de água lateral. Já no de madeira, a absorção total foi superior após 2 horas, mas igualou-se à absorção superficial após 24 horas.
\end{abstract}

Palavras-chave: materiais lignocelulósicos, painéis aglomerados, propriedades físicas.

\section{Physical Properties of Commercial Particle-boards Made of Sugarcane Bagasse and Wood}

\begin{abstract}
The physical properties of commercial particle-boards made of sugar cane bagasse and wood were studied. Thickness swelling and water absorption tests were conducted in order to compare the materials and their types of absorption: total, lateral and superficial. Tests for comparison between particle-boards made of different materials showed that sugar cane bagasse particle-boards presented higher water absorption, but lower thickness swelling. For sugar cane bagasse particle-board, the highest value was found for lateral water absorption. For wood particle-board, the total absorption was highest after 2 hours of immersion, but it became equal to the superficial absorption after 24 hours.
\end{abstract}

Keywords: lignocellulosic materials, particle-boards, physical properties.

\section{INTRODUÇÃO}

Os painéis aglomerados são altamente empregados na confecção de móveis e na construção civil. No Brasil, a primeira unidade industrial destes painéis foi instalada no Paraná, em 1966, e, desde então, várias outras indústrias surgiram pelo País (Iwakiri et al., 2001). Existe um potencial significativo para a produção destes painéis no Brasil devido à alta disponibilidade de insumos para o processo produtivo (Noce et al., 2008).

O painel de madeira aglomerada é formado por pequenas partículas de madeira e/ou de outros materiais lignocelulósicos aglutinadas com 
um adesivo sintético e consolidadas sob alta pressão e temperatura. Apesar de a madeira ser a principal matéria-prima para produção de painéis aglomerados, diversos países têm buscado a sua substituição, pelo menos parcial, por resíduos agrícolas. Assim, atualmente, pesquisadores vêm mostrando a viabilidade do emprego de resíduos de diversas origens para a produção de aglomerados, pois, além de contribuir para o atendimento da demanda de painéis, gera materiais que preservam os recursos naturais e oferece um destino adequado aos resíduos (Mendes et al., 2009).

Segundo Widyorini et al. (2005), dentre as inúmeras matérias-primas não lenhosas que apresentam potencial para esta finalidade, o bagaço de cana é considerado o mais promissor para substituição da madeira, já que estes materiais lignocelulósicos apresentam características químicas e anatômicas similares. Aliado a este fato, Basttinelli et al. (2008) apontam o Brasil como maior produtor de cana-de-açúcar do mundo, sendo este resíduo pouco explorado.

Na China, por outro lado, o quadro atual é bastante diferente. A criação do "Programa de Proteção das Florestas Anuais", somada com a demanda crescente por produtos reconstituídos e, ainda, com a diminuição da oferta de madeira, incentivaram o governo do país a desenvolver programas que encorajem a substituição da madeira por outros produtos para diversos fins ao longo dos últimos 10 anos. A produção de painéis reconstituídos de resíduos agrícolas se apresenta como o principal interesse para utilização desses resíduos (Xu et al., 2004). Wei et al. (2004) afirmam que bagaço de cana também já é bastante utilizado para produção comercial de painéis aglomerados na China.

No Brasil, a viabilidade técnica de utilização do bagaço de cana para produção de painéis aglomerados já vem sendo estudada por diversos pesquisadores em escala laboratorial (Okino et al., 1997; Teixeira et al., 1997; Basttinelli et al., 2008; Mendes et al., 2009). No entanto, estudos comparativos entre painéis aglomerados comerciais de bagaço de cana-de-açúcar e de madeira são escassos na literatura. Resultados dessa natureza seriam interessantes para dar suporte e informação aos potenciais produtores brasileiros de aglomerados de bagaço de cana-de-açúcar sobre a qualidade desses produtos.

Dentre os estudos de interesse para a qualificação de painéis aglomerados, o conhecimento das propriedades físicas é fundamental. Com a análise da capacidade de absorção de água do ambiente, podese recomendar a utilização do painel em condições de umidade severas ou ambientes mais amenos. Além disso, é interessante verificar a resistência das faces dos painéis à umidade do ambiente, uma vez que elas são as áreas do painel mais expostas durante o seu uso.

Estudos para verificar se painéis aglomerados de bagaço de cana-de-açúcar já comercializados na China apresentam qualidade melhor ou similar a painéis de madeira comercializados no Brasil seriam de grande valia para incentivar a utilização de resíduos agrícolas na produção de painéis. Os objetivos desse trabalho foram: 1) comparar painéis aglomerados comerciais de bagaço de cana-deaçúcar com painéis aglomerados comerciais de madeira quanto às propriedades físicas; 2) verificar se as obstruções das laterais e das faces dos painéis diminuem a absorção de água do ambiente e interferem nos resultados dos testes físicos.

\section{MATERIAL E MÉTODOS}

Foram utilizados painéis aglomerados MDP comerciais com as seguintes dimensões: $244 \times 122 \times 1,5 \mathrm{~cm}$. Os tratamentos comparados corresponderam a painéis de bagaço de cana, proveniente da China e de madeira de Eucalyptus sp. em mistura com madeira de Pinus sp., produzidos no Brasil, sendo ambos vendidos comercialmente. As variáveis de processamento não foram fornecidas pelas indústrias. Dessa forma, os painéis comerciais foram comparados considerando-se todas as possíveis diferenças nas variáveis de processamento.

Foram amostrados 3 painéis de cada tipo, sendo obtidos 15 corpos de prova de cada painel com dimensões nominais $152 \times 152 \times 15 \mathrm{~mm}$. O material foi levado para sala de climatização e mantido à temperatura de $20{ }^{\circ} \mathrm{C}$ e umidade relativa de $65 \%$ até atingir massa constante.

Anteriormente à realização dos testes físicos, cinco corpos de prova de cada painel receberam 
uma camada de parafina nas laterais, enquanto que outros cinco receberam parafina nas superfícies. $\mathrm{O}$ restante dos corpos de prova não recebeu nenhuma obstrução com parafina.

Em seguida, os testes físicos de absorção de água (após 2 e 24 horas de imersão), inchamento em espessura ( 2 e 24 horas de imersão) e densidade foram realizados de acordo com a norma ASTM D 1037 (2006). Para comparação entre os painéis avaliados sem obstrução de parafina (madeira e bagaço de cana-de-açúcar), incialmente foi realizada uma análise de covariância com o intuito de eliminar o efeito da densidade sobre as propriedades físicas. Em seguida, foi empregado um delineamento experimental inteiramente casualizado composto por dois tratamentos e três repetições. Já para comparação entre as amostras submetidas a diferentes condições de obstrução com parafina, o delineamento experimental foi inteiramente casualizado e as seguintes condições foram comparadas pelo teste de Scott-Knott a 5\% de significância: absorção total, superficial e lateral (Figura 1a,b), com 3 repetições cada.

\section{RESULTADOS E DISCUSSÃO}

\subsection{Painéis de bagaço de cana-de-açúcar em comparação com painéis de madeira}

Os painéis aglomerados comerciais produzidos com diferentes materiais lignocelulósicos apresentaram densidade média aparente estatisticamente diferente (Figura 2). Este resultado pode exercer influência sobre a absorção de água e inchamento em espessura dos painéis.

Os resultados das análises de covariância para verificação da influência da densidade aparente sobre as propriedades físicas dos painéis nas condições sem obstrução de parafina estão expostos na Tabela 1.

As propriedades que apresentaram influência da densidade aparente sobre seus valores foram transformadas de acordo com os modelos apresentados.

Os parâmetros estatísticos para testes físicos realizados para comparação entre os painéis aglomerados comerciais sem obstrução de parafina estão apresentados na Tabela 2.

Os valores de inchamento em espessura 2-24 horas e absorção de água 2-24 horas estão apresentados na Figura 3.

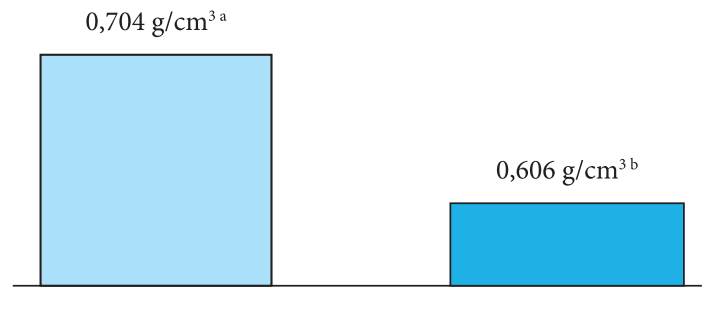

F calculado $=35,951$ significativo;

Coeficiente de variação $=6,83 \%$

$\square$ Madeira $\square$ Bagaço de cana

Figura 2. Densidade aparente média dos painéis aglomerados.

Figure 2. Average apparent density of the particleboards.
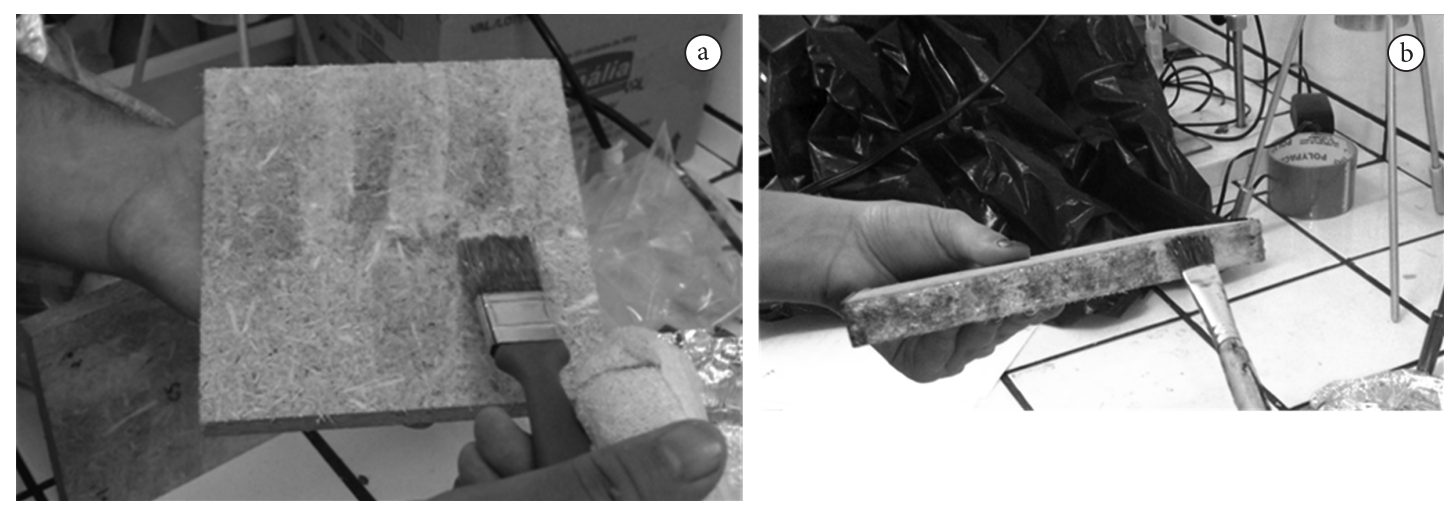

Figura 1. a) Aplicação de parafina na superfície; b) Aplicação de parafina na lateral. Figure 1. a) Paraffin application in the surface; b) Paraffin application in the lateral. 
Tabela 1. Resultados das análises de covariância.

Table 1. Results of covariance analysis.

\begin{tabular}{|c|c|c|c|}
\hline Condição & Propriedade & Fc & Modelo \\
\hline \multirow{4}{*}{ Sem obstrução com parafina } & AA_2h & $34,114^{*}$ & AA_2h $=40,33-39,64^{*} \mathrm{D}$ \\
\hline & AA_24h & $3,421^{\text {ns }}$ & - \\
\hline & IE_2h & $1,415^{\mathrm{ns}}$ & - \\
\hline & IE_24h & $15,655^{\star}$ & IE_24h $=-23,07+58,32 * \mathrm{D}$ \\
\hline
\end{tabular}

Tabela 2. Parâmetros estatísticos dos testes para comparação entre os painéis comerciais.

Table 2. Statistical parameters of the tests for comparison between commercial panels.

\begin{tabular}{lcc}
\multicolumn{1}{c}{ Propriedade } & F calculado & Coeficiente de variação (\%) \\
\hline Absorção de água_2h & $40,362^{*}$ & 4,52 \\
Absorção de água_24h & $22,876^{*}$ & 8,29 \\
Inchamento em espessura_2h & $1,064^{\text {ns }}$ & 18,18 \\
Inchamento em espessura_24h & $40,355^{*}$ & 7,59 \\
\hline
\end{tabular}

$\mathrm{e}^{*}$ : valor de $\mathrm{F}$ calculado significativo.

Foi possível observar que houve diferença estatística significativa para os testes de absorção de água após 2 e 24 horas e inchamento em espessura após 24 horas. Os maiores valores de absorção de água foram observados para os painéis de bagaço de cana. Os resultados encontrados neste trabalho podem ser explicados pela barreira física à entrada de água causada pela diminuição dos espaços vazios entre as partículas nos painéis de madeira que apresentam maior densidade. Além disso, resíduos agrícolas usualmente apresentam maiores teores de hemiceluloses em relação a resíduos de madeira. Esses componentes apresentam mais sítios de ligação química com a água, podendo resultar em maior absorção de água em painéis feitos com esses materiais (Li et al., 2011).

Melhoras no processo produtivo poderiam ser realizadas com o intuito de diminuir a absorção de água dos painéis de bagaço de cana, tais como utilização de pré-tratamento das partículas de resíduos agrícolas a vapor para degradação de hemiceluloses, reduzindo o número de sítios higroscópicos (Li et al., 2011) e aplicação de materiais de revestimento que funcionam como uma barreira física à penetração de água no painel (Nemli, 2008).

$\mathrm{O}$ maior inchamento em espessura verificado após 24 horas para os painéis de madeira possivelmente foi influenciado pela maior densidade

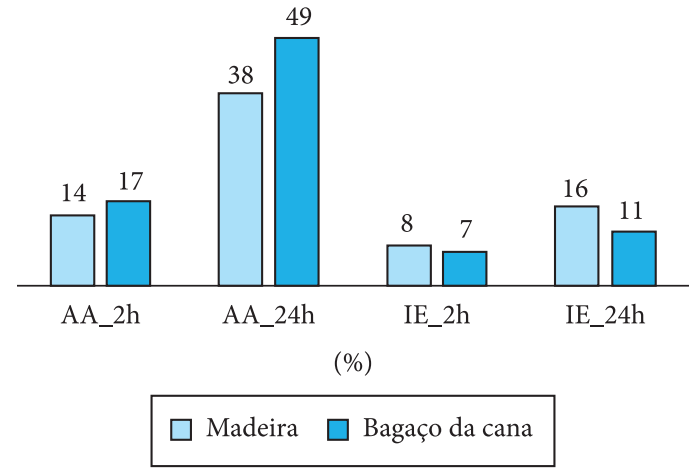

Figura 3. Comparação entre as propriedades físicas dos painéis aglomerados sem obstrução de parafina.

Figure 3. Comparison between physical properties of the particleboards without paraffin obstruction.

desses produtos, uma vez que ocorre maior liberação das forças de compressão geradas durante o processo de prensagem em produtos de maior densidade (efeito springback).

Mendes et al. (2009) encontraram valores de absorção de água após 24 horas de 95,78 e 104,56\% para painéis aglomerados de $0,70 \mathrm{~g} / \mathrm{cm}^{3}$ com $1 \%$ de parafina produzidos com Eucalyptus urophylla de 7 e 12 anos de idade, respectivamente. Já Batista et al. (2007) avaliaram as propriedades físicas de painéis aglomerados de três camadas de Pinus elliottii Engelm. produzidos com $1 \%$ de parafina e 
6\% de ureia-formaldeído e encontraram valores de absorção de água de 150,6 e 168,9\% após 2 e 24 horas, respectivamente. Em ambos os estudos mencionados acima, os testes foram realizados segundo a mesma norma utilizada neste trabalho. Estes valores obtidos da literatura são consideravelmente superiores em relação aos encontrados neste trabalho para painéis aglomerados de madeira e bagaço de cana-deaçúcar, demonstrando que melhoras são necessárias na produção de painéis em escala laboratorial para comparação com painéis comerciais.

Os resultados encontrados para inchamento em espessura para os dois painéis aglomerados comerciais deste trabalho foram inferiores aos apresentados por Mendes et al. (2010). Os autores, estudando as propriedades físicas de aglomerados de bagaço de cana em associação com madeira de eucalipto segundo a mesma norma utilizada neste trabalho, encontraram valores médios de inchamento em espessura para 2 e 24 horas de imersão em água de 9,15 e $18,16 \%$, respectivamente.

A norma CS 236-66 (Commercial..., 1968) estabelece um valor máximo de inchamento em espessura após 24 horas de imersão em água de 35\% para painéis aglomerados destinados a uso interior. Dessa forma, os resultados apresentados por ambos os painéis aglomerados comerciais apresentam-se aptos para utilização em locais de alta umidade.

\subsection{Painéis de bagaço de cana-de-açúcar: comparação entre as diferentes condições}

Os parâmetros estatísticos dos testes físicos para comparação entre os diferentes tipos de absorção dos painéis de bagaço de cana-de-açúcar estão apresentados na Tabela 3. Foi possível observar diferenças estatísticas significativas entre os tipos de absorção para todas as propriedades físicas estudadas.

Os valores médios das propriedades físicas dos painéis aglomerados comerciais de bagaço de canade-açúcar e a comparação entre tratamentos pelo teste de Scott-Knott estão apresentados na Figura 4.

De forma geral, a obstrução dos corpos de prova com parafina não resultou em diminuição da absorção de água, uma vez que a absorção total foi estatisticamente igual à absorção lateral após 2 horas de imersão e inferior a ambas as absorções laterais e superficiais após 24 horas de imersão.

Os valores de inchamento em espessura após 24 horas das amostras submetidas à absorção de água lateral foram superiores em relação aos valores das amostras submetidas à absorção de água total e superficial. A entrada de água somente pelas laterais das amostras pode ter resultado em maior

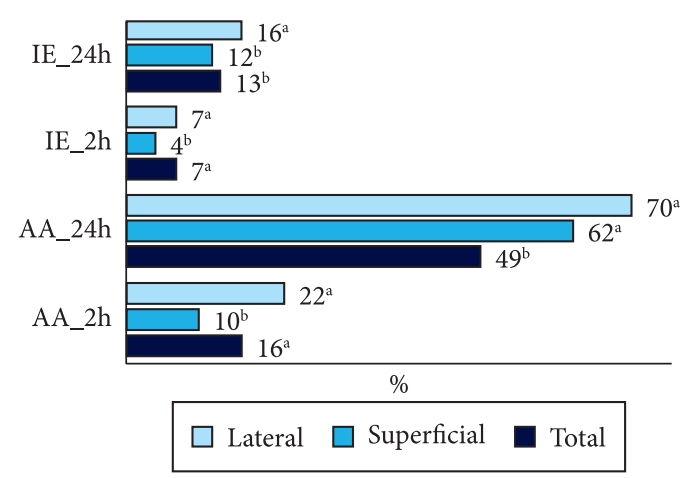

Figura 4. Valores médios das propriedades físicas das amostras retiradas do painel aglomerado de bagaço de cana-de-açúcar e submetidas aos diferentes tratamentos. Figure 4. Average values of the physical properties of the samples obtained from the sugar cane bagasse particleboard and submitted to different treatments.

Tabela 3. Parâmetros estatísticos dos testes para comparação entre os tipos de absorção do aglomerado de bagaço de cana-de-açúcar.

Table 3. Statistical parameters of the tests for comparison between the absorption types of the sugar cane particleboard.

\begin{tabular}{lcc}
\multicolumn{1}{c}{ Propriedade } & F calculado & Coeficiente de variação (\%) \\
\hline Absorção de água_2h & $6,368^{*}$ & 34,63 \\
\hline Absorção de água_24h & $6,307^{*}$ & 14,23 \\
Inchamento em espessura_2h & $9,147^{*}$ & 25,06 \\
Inchamento em espessura_24h & $19,209^{*}$ & 8,68 \\
\hline
\end{tabular}

$\mathrm{e}^{*}$ : valor de F calculado significativo. 
Tabela 4. Parâmetros estatísticos dos testes para comparação entre os tipos de absorção do aglomerado de madeira. Table 4. Statistical parameters of the tests for comparison between the absorption types of the wood particleboard.

\begin{tabular}{lcc}
\multicolumn{1}{c}{ Propriedade } & F calculado & Coeficiente de variação (\%) \\
\hline Absorção de água_2h & $12,234^{*}$ & 8,58 \\
Absorção de água_24h & $12,363^{*}$ & 12,40 \\
Inchamento em espessura_2h & $6,109^{*}$ & 20,00 \\
Inchamento em espessura_24h & $0,240^{\text {ns }}$ & 12,34 \\
\hline
\end{tabular}

$\mathrm{e}^{\star}$ : valor de F calculado significativo; $\mathrm{e}^{\mathrm{ns}}$ : valor de F calculado não significativo.

liberação das forças de compressão geradas durante a prensagem do painel.

Até duas horas de imersão em água, as superfícies do painel pareciam ser mais resistentes à absorção de água, o que também resultou em um baixo valor de inchamento em espessura. As partículas menores e mais compactadas que compõem a superfície em relação ao miolo do painel podem ter contribuído para esse resultado pela formação de uma barreira física à entrada de água. No entanto, a absorção superficial se igualou à absorção lateral após 24 horas.

\subsection{Painéis de madeira: comparação entre as diferentes condições}

Os parâmetros estatísticos dos testes físicos para comparação entre os diferentes tipos de absorção dos painéis de madeira estão apresentados na Tabela 4. Foi possível observar diferenças estatísticas significativas entre os tipos de absorção para todas as propriedades físicas estudadas.

Os valores médios das propriedades físicas dos painéis de madeira aglomerada e a comparação entre tratamentos pelo teste de Scott-Knott estão apresentados na Figura 5.

Após 2 horas de imersão em água, a absorção de água total das amostras do painel aglomerado de madeira foi superior às absorções laterais $\mathrm{e}$ superficiais, indicando que, até esse instante, a obstrução das amostras com parafina contribui para reduzir o valor desta propriedade. O maior valor de absorção de água foi acompanhado pelo maior valor de inchamento em espessura após 2 horas de imersão das amostras de painel sem aplicação da parafina.

Após 24 horas, porém, a absorção superficial de água das amostras de painel aglomerado foi estatisticamente superior em relação às absorções

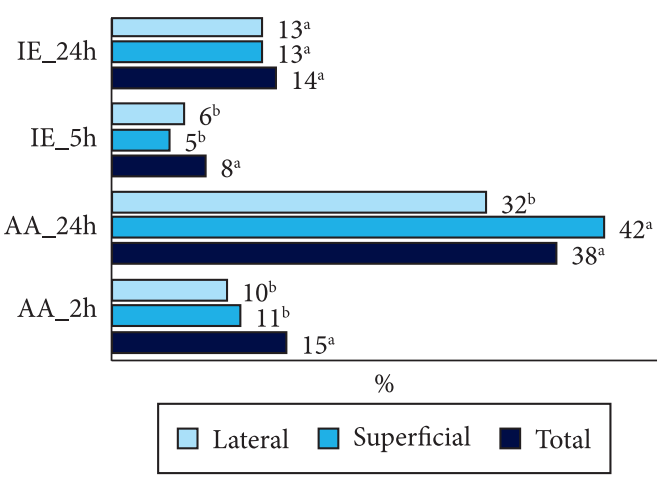

Figura 5. Valores médios das propriedades físicas das amostras retiradas do painel aglomerado de madeira $\mathrm{e}$ submetidas aos diferentes tratamentos.

Figure 5. Average values of the physical properties of the samples obtained from the wood particleboard and submitted to different treatments.

totais e laterais. No entanto, após 24 horas, a absorção superficial se igualou estatisticamente à absorção total, indicando, possivelmente, pouca resistência da superfície desses painéis a condições de alta umidade. A absorção lateral das amostras foi inferior após 24 horas de imersão em água. Apesar das diferenças nas absorções após esse período de tempo, não foi verificada diferença estatística significativa entre os inchamentos em espessura.

\section{CONCLUSÕES}

1) Nos testes de comparação entre painéis de bagaço de cana e painéis de madeira foi observado que os primeiros apresentaram maiores valores de absorção de água, mas menores inchamentos em espessura. 
2) Para o painel aglomerado de bagaço de cana-deaçúcar, foi observado que amostras com as laterais susceptíveis à entrada de água apresentam maior inchamento em espessura após 24 horas. Para absorção de água e inchamento em espessura após 2 horas, foram observados menores valores para as amostras com as superfícies expostas e laterais vedadas. Para absorção de água após 24 horas, as amostras vedadas nas laterais e nas superfícies apresentaram-se estatisticamente iguais.

3) Para o painel aglomerado de madeira, verificou-se que as amostras com as superfícies susceptíveis à entrada de água apresentaram maior absorção de água após 24 horas. Para o inchamento em espessura após 24 horas, as amostras apresentaramse estatisticamente iguais. A absorção de água e inchamento em espessura após 2 horas foi maior em amostras sem obstrução de parafina.

4) Os resultados e comparações obtidos neste trabalho poderiam ter sido mais consistentes se as variáveis de produção dos painéis, tais como tipo e proporção de adesivo, especificações do ciclo de prensagem e geometria de partícula tivessem sido fornecidas pelas empresas produtoras dos painéis. Apesar de desconhecidas, todas essas fontes de variação certamente influenciaram diretamente os resultados.

\section{AGRADECIMENTOS}

Os autores agradecem ao CNPq, à Fapemig e à Capes.

\section{STATUS DA SUBMISSÃO}

Recebido: 22/12/2010

Aceito: 11/06/2011

Resumo publicado online: 25/06/2011

Artigo completo publicado: 30/06/2011

\section{AUTOR (ES) PARA CORRESPONDÊNCIA}

\section{Cecília de Souza Freire}

Setor de Ciência e Tecnologia da Madeira, Unidade Experimental de Produção de Painéis de Madeira, Departamento de Ciências Florestais DCF, Universidade Federal de Lavras - UFLA, CP 3037, CEP 37200-000, Lavras, MG, Brasil e-mail: ceciliafreire540@hotmail.com

\section{Mário Vanoli Scatolino}

Setor de Ciência e Tecnologia da Madeira, Unidade Experimental de Produção de Painéis de Madeira, Departamento de Ciências Florestais DCF, Universidade Federal de Lavras - UFLA, CP 3037, CEP 37200-000, Lavras, MG, Brasil e-mail: mario_paraiso@hotmail.com

\section{REFERÊNCIAS}

American Society for Testing - ASTM. ASTM D103706a: Standard test methods for evaluating Properties of Wood-Base Fiber and Panel Materials. Philladelphia: ASTM; 2006.

Basttinelli, RAG, Marcilio C, Lahr FAR. Emprego do bagaço de cana-de-açúcar (Saccharum officinarum) e das folhas caulinares do bambu da espécie Dendrocalamus giganteus na produção de chapas de partículas. Revista Minerva 2008; 5(3):297-305.

Batista DC, Brito EO, Setubal VG, Góes LG. Fabricação de aglomerados de três camadas com madeira de Pinus elliottii Emgelm. e casca de Eucalyptus pellita F. Muell. Cerne 2007; 13(2):178-187.

Commercial Standard. CS 236-66: Mat formed wood particleboard. 1968.

Iwakiri S, Silva JRM, Matoski SLS, Leonhadt G, Caron J. Produção de chapas de madeira aglomerada de cinco espécies de pinus tropicais. Floresta e Ambiente 2001; 8(1):137-142.

Li X, Cai Z, Winandy JE, Basta AH. Effect of oxalic acid and steam pretreatment on the primary properties of UF-bonded rice straw particleboards. Industrial Crops and Products 2011; 33:665-669. http://dx.doi. org/10.1016/j.indcrop.2011.01.004

Mendes LM, Iwakiri S, Mori FA, Guimarães Júnior JB, Mendes RF. Eucalyptus urophylla stands wood utilization at two different ages for production of particleboard panels. Cerne 2009; 15(3):288-294.

Mendes RF, Mendes LM Abranches RAS, Santos RC, Guimarães Junior JB. Painéis aglomerados produzidos com bagaço de cana em associação com madeira de eucalipto. Scientia Forestalis 2010; 38(86):285-295.

Nemli G. Factors affecting some quality properties of the decorative surface overlays. Journal of Materials Processing Technology 2008; 95(1):218-223. http:// dx.doi.org/10.1016/j.jmatprotec.2007.05.001

Noce R, Silva ML, Souza AL, Silva OM, Mendes LM, Carvalho RMMA et al. Competitividade do Brasil no mercado internacional de aglomerado. Revista Árvore 2008; 32(1):113-118. http://dx.doi.org/10.1590/S010067622008000100013 
Okino EYA, Andahur JPV, Santana MAE, Souza MR. Resistência físico-mecânica de chapas aglomeradas de bagaço de cana-de-açúcar modificado quimicamente. Scientia Forestalis 1997; 52:35-42.

Teixeira DE, Costa AF Santana MAE. Aglomerados de bagaço de cana-de-açúcar: resistência natural ao ataque de fungos apodrecedores. Scientia forestalis 1997; 52:29-34.

Wei YA, Lu JJ, Sun WD. New development in sugarbased products and cane by-products utilization in
China. Sugar Tech 2004; 6(4):281-284. http://dx.doi. org/10.1007/BF02942509

Widyorini R, Xu J, Umemura K, Kawai S. Manufacture and properties of binderless particleboard from bagasse I: effects of raw material type, storage methods, and manufacturing process. Journal of Wood Science 2005; 51(6):648-654. http://dx.doi.org/10.1007/s10086-0050713-Z

Xu BX, Zhou D, Wu Q, Vloski RP. Agri-Based composites in China: opportunities and challenges. Forest Products Journal 2004; 54(5):8-15. 